\title{
Age at menarche and cognitive functioning
}

\author{
JILL RIERDAN and ELISSA KOFF \\ Wellesley College, Wellesley, Massachusetts
}

\begin{abstract}
This investigation sought to determine if a relationship between rate of pubertal development and cognitive styles, previously observed in early adolescents, holds over time. In contrast to reports that early and late maturers, as early adolescents, have different patterns of cognitive abilities, two studies failed to confirm a relationship between maturation rate, indexed by age at menarche, and (1) spatial ability, (2) verbal fluency, or (3) relative degree of spatial versus verbal fluency in late-adolescent women. The results suggest a developmental instability of maturation-rate/cognition relationships, at least among females.
\end{abstract}

Recent studies (Petersen, 1976; Waber, 1976, 1977) have argued that the frequently observed male superiority in spatial ability and female superiority in verbal ability (Maccoby \& Jacklin, 1974) can be attributed, at least in part, to biological phenomena. In particular, hormonal status has been implicated, because sex differences in cognition often emerge or increase dramatically with the onset of puberty, a time of greatly increased sex-hormone levels. Between-sex differences in cognition have been explained in terms of within-sex differences in hormonal status, as indexed by degree of physical androgyny (Petersen, 1976) and by rate of sexual maturation (Waber, 1976, 1977).

Waber $(1976,1977)$ administered a series of verbal and spatial tasks to a group of 10-13-year-old girls and 13-16year-old boys. The male sample was older, to take into account the finding that boys typically attain puberty several years later than girls (Tanner, 1978). Subjects whose chronological age was at least one standard deviation below the mean age associated with their stage of physical development (using a variety of measures of maturation) were classified as early maturers; those with a chronological age at least one standard deviation above the mean age associated with their stage of physical development were classified as late maturers.

Waber $(1976,1977)$ found, for both sexes, that late maturers had higher spatial scores than early maturers. This seemed to parallel the prototypic finding that males, who are relatively late maturers, receive higher spatial scores than females, who are relatively early maturers. In contrast, maturation rate was not related to verbal ability. Sex differences in cognition-independent of maturation level-also were not significant. Additional analyses examined cognitive differences within, as opposed to between, early and late maturers and indicated that late maturers, regardless of sex, received

Preparation of this manuscript was supported, in part, by Grant HD 16034 from the National Institute of Child Health and Human Development. We thank Margaret Stubbs for assistance in the data analysis. The authors' mailing address is: Center for Research on Women, Wellesley College, Wellesley, MA 02181. higher scores on spatial than on verbal tasks, whereas early maturers showed the opposite pattern. On the basis of these findings, Waber proposed that the frequently observed pattern of sex differences in cognitive ability (Maccoby \& Jacklin, 1974) might be moderated by sex differences in maturation rate, with this latter variable correlated both with gender and with cognitive style. If this interpretation is valid, Waber's failure to obtain the typical pattern of sex differences in cognition in her own research is readily explained: by equating the maturational status of her subjects (there were equal numbers of early- and late-maturing boys and girls), she was in fact controlling for the very variable that theoretically accounts for the sex differences in cognition found when random groups of males and females, typically differing significantly in rate of maturation, are tested.

One interesting question that Waber's $(1976,1977)$ data raise is whether the type of relationship she postulates between maturation rate and cognitive functioning holds over time. A variety of physical changes associated with puberty are occurring throughout the 10-16-year range (Tanner, 1978), as are changes in cognitive functioning (Inhelder \& Piaget, 1958). Thus, psychobiological correlations significant in early adolescence may not necessarily obtain in late adolescence, by which time changes in a variety of aspects of biological and psychological functioning have occurred. Before generalizations can be made about differences in cognition associated with differential rates of maturation, these relationships must be assessed in subjects who have attained both physical and cognitive maturity. Only then can we determine whether these relationships are reliable phenomena or transitory characteristics of developing adolescents.

A second question arising from Waber's $(1976,1977)$ data concerns the varieties of cognitive functioning that may be related to maturation rate. Does rate of maturation affect only spatial ability, or does it affect aspects of verbal functioning as well?

The most obvious and efficacious way to address the stability question would be with a longitudinal design. 
This would be extremely time-consuming and expensive, since it would require repeated testing of children from about age 10 through 18 years to obtain an adequate sample of early, average, and late maturers, assessed in early and late adolescence. An alternative approach, which is reported here, would be to study a group of subjects who have attained sexual and cognitive maturity and who could accurately be classified, retrospectively, in terms of rate of maturation. The difficulty presented by this latter approach is in finding a reliable and valid method of determining rate of physical maturation retrospectively for men and women. One way to do this for women is to classify them according to age at menarche, or first menstruation. Memory about this particular marker of maturation has proved to be quite reliable over time, having been shown to be recalled accurately as long as 30 years after the event (Bean, Leeper, Wallace, Sherman, \& Jagger, 1979; Damon \& Bajema, 1974). Unfortunately, no comparable marker exists for males. Accordingly, in this research, we assessed the generality over time of Waber's $(1976,1977)$ maturation-cognition hypothesis with females only.

In the two studies reported here, spatial (Study 1) and verbal and spatial tasks (Study 2) were administered to late-adolescent/early-adult women. The subjects were classified on a continuum of rate of maturation according to recalled age at menarche. Spatial ability was assessed with the Group Embedded Figures Test (GEFT; Witkin, Oltman, Raskin, \& Karp, 1971), and verbal ability, or more specifically, verbal fluency, by the Digit Symbol (DS) subtest of the Wechsler Adult Intelligence Scale-Revised (WAIS-R; Wechsler, 1981). These measures, or their analogues, frequently are employed in studies of sex differences in cognitive abilities (e.g., Waber, 1976, 1977, used the DS of the Wechsler Intelligence Scale for Children and a modified version of the GEFT). On the assumption that Waber's data were valid, and that the patterns of cognitive skills she observed were stable, we predicted that late-maturing females would perform better than early-maturing females on the GEFT, and better on the GEFT than on the DS subtest. We also expected early-maturing females to perform better on the DS than on the GEFT.

\section{STUDY 1}

\section{Method}

Subjects. Eighty-four college women (mean age $=19.84$ years) participated voluntarily as subjects. Ages at menarche ranged from 9.58 to 17.75 years, with a mean of 12.87 years.

Procedure. The subjects were tested individually by a female experimenter. They answered questions regarding age at menarche and other demographic variables, and then completed the GEFT.

\section{Results}

A correlational analysis performed on ages at menarche and the GEFT (with scores ranging from 1 to 18 ; mean $=13.40)$ failed to reach significance $(r=0.02$, Pearson product-moment). In the event that a nonlinear type of relationship might be obscured by this treatment of the data, we trichotomized the age-at-menarche variable and conducted an analysis of variance (ANOVA) on the GEFT scores for the three maturational rate groups. According to standard norms, which place the average age at menarche at 12.8 -13.2 years ( \pm 12 months) (Tanner, 1978), the subjects who had attained menarche before the age of 12 years $(\mathrm{N}=22)$ were classified as early maturers, those who had attained menarche between the ages of 12 and 13.9 years $(N=47)$ were classified as average maturers, and those who had attained menarche after the age of 14 years $(\mathrm{N}=15)$ were classified as late maturers. The ANOVA revealed no significant differences among the three menarcheal age groups $[F(2,81)=.54]$. Mean GEFT scores for the three age groups were 13.54, 13.11, and 14.40.

\section{STUDY 2}

\section{Method}

Subjects. Sixty right-handed (Briggs \& Nebes, 1975) college women (mean age $=20.07$ years) participated in this study. Ages at menarche ranged from 9.83 to 18.17 years, with a mean of 12.81 years.

Procedure. The subjects were tested individually by a female experimenter. After they had responded to questions about age at menarche and other demographic variables, the cognitive tests were administered. Order of administration of the two measures was counterbalanced, with half the subjects beginning with the GEFT and half with the DS subtest.

\section{Results}

Scores on the GEFT ranged from 1 to 18 , with a mean of 13 , and scores on the DS subtest ranged from 50 to 90 , with a mean of 71.59 . These means exceed those that have been reported previously for women for both tests (for GEFT, Witkin et al., 1971; for DS, Wechsler, 1981). However, since scores were neither homogeneous nor so high as to constitute a ceiling effect, evaluation of a relationship between age at menarche and cognition remained valid.

As in Study 1, Pearson product-moment correlations were determined for age at menarche and GEFT scores $(\mathrm{r}=0.13)$ and for age at menarche and DS scores $(\mathrm{r}=$ $-0.07)$. Neither correlation was significant.

One-way ANOVAs were then computed to determine differences among the early-maturing $(\mathrm{N}=16)$, average $(\mathrm{N}=36)$, and late-maturing $(\mathrm{N}=8)$ women on the GEFT and DS subtests. Again, no significant differences among the three menarcheal age groups emerged [for GEFT, $F(2,57)=1.03$; for DS, $F(2,57)=0.58]$. Mean GEFT scores for the three groups were 12.13, 13.00, and 14.75; mean DS scores were $73.13,64.72$, and 73.63 .

Finally, GEFT and DS scores were transformed to $\mathrm{Z}$ scores to permit analysis of the intrasubject relationship of spatial to verbal ability. Difference scores were obtained by subtracting the $Z$ score for DS from the $Z$ score for GEFT. The difference scores were then correlated with age at menarche, but no significant relationship was evident $(r=.16)$. Difference scores were 
also subjected to a one-way ANOVA with three menarcheal age groups. No differences among the three menarcheal age groups were obtained $[\mathrm{F}(2,57)=1.68]$, with the means being $-0.23,0.25$, and 0.01 .

\section{DISCUSSION}

Our general framework for interpreting the results of the present studies and those of Waber $(1974,1977)$ and others (e.g., Berenbaum \& Resnick, 1982; Petersen, 1976) is the literature concerning the long-term impact of rate of physical maturation on women's psychological functioning. Clausen (1975) and Livson and Peskin (1980) have reviewed this research and concluded that, from childhood to adulthood, instability in biopsychological relationships seems to be the norm. Significant biopsychological correlations observed in early-adolescent girls may be absent or may reverse direction when these same girls are tested as adults. Accordingly, the fact that our data and those of two other studies using retrospective age at menarche as an index of maturation (Kennedy, 1971/1972; Strauss \& Kinsbourne, 1981) failed to observe a cognition-maturation relationship in adult women such as had previously been observed with young adolescents (Waber, 1976, 1977) is not surprising. Earlymaturing females may indeed show patterns of cognitive functioning different from those shown by their later-maturing peers when they are young adolescents, whereas these same early- and late-maturing females may be indistinguishable cognitively as adults. Clearly, what is needed to reach firm conclusions about maturation-cognition relationships is a long-term developmental study using a variety of markers denoting pubertal status, in which an array of cognitive tasks believed to be sensitive to variations in brain organization are administered, and in which variables that have been shown to moderate maturation-rate/psychological relationships, such as social class (Clau sen, 1975), are controlled.

Because only women were studied in the present investigation, our conclusions regarding developmental patterns of maturation-rate/cognition relationships must be restricted to females. This is important to note in the context of previous research assessing biopsychological relationships (Clausen, 1975; Livson \& Peskin, 1980). The impact of rate of maturation on psychological functioning tends to be more stable over time for men than for women; accordingly, developmental patterns of maturation-cognition relationships may differ for women and men, with males perhaps evidencing the cognitive patterns observed by Waber $(1976,1977)$ in late as well as early adolescence.

The use of a retrospective measure of maturation rate in the present investigation, namely, age at menarche, may be regarded as a limitation. Although the reliability of long-term memory has been questioned, the time interval in the study, between menarche and the report of its occurrence (an average of 6 years), is unlikely to be associated with significant memory distortion (Yarrow, Campbell, \& Burton, 1970). Furthermore, the accuracy of women's memories of age at menarche has been validated in a number of investigations (Bean et al., 1970; Damon \& Bajema, 1974). Thus, the use of menarcheal age as a retrospective measure of maturation rate is unlikely to limit the validity of our findings.

We suggested, above, that future investigations should make use of more than one measure of spatial and verbal functioning. It may be that maturation-cognition relationships in late adolescence will be found with cognitive tasks not used in the two studies reported here. At present, though, it is important that such a relationship was not found on the two widely used tasks employed in this investigation.
In summary, the present study assessed the generality of Waber's $(1976,1977)$ maturation-rate/cognition hypothesis in a sample of late-adolescent women and failed to confirm a relationship between maturation rate, indexed by age at menarche, and (1) spatial ability, (2) verbal fluency, or (3) relative degree of spatial versus verbal fluency. These results suggest that a relationship between maturation rate and cognition may be limited in power and generality. Furthermore, specification of the parameters of maturation-rate/cognition relationships is especially important, since the question of inherent cognitive differences within and between groups of women and men is of educational and social as well as scientific significance.

\section{REFERENCES}

Bean, J. A., Leeper, J. D., Wallace, R. B., Sherman, B. M., \& JAGGER, H. (1979). Variations in reporting of menstrual histories. American Journal of Epidemiology, 109, 181-185.

Berenbaum, S. A., \& Resnick, S. (1982). Somatic androgyny and cognitive abilities. Developmental Psychology, 18, 418-423.

Briggs, C. G., \& Nebes, R. D. (1975). Patterns of hand preference in a student population. Cortex, 11, 230-238.

Clausen, J. A. (1975). The social meaning of differential physical and sexual maturation. In S. E. Dragastin \& G. H. Elder (Eds.), Adolescence in the life cycle: Psychological change and social context. New York: Wiley.

Damon, A., \& Bajema, C. J. (1974). Age at menarche: Accuracy of recall after 39 years. Human Biology, 46, 381-384.

INHELder, B., \& PiAGet, J. (1958). The growth of logical thinking from childhood to adolescence. New York: Basic Books.

Livson, N., \& Peskin, H. (1980). Perspectives on adolescence from longitudinal research. In J. Adelson (Ed.), Handbook of adolescent psychology. New York: Wiley.

KENNEDY, S. J. L. (1972). The relationship between the onset of adolescent physical maturation and features of cognitive style as expressed in adults (Doctoral dissertation, Wayne State University, 1971). Dissertation Abstracts International, 32, 6620B.

MaccoBy, E. E., \& JaCkLin, C. N. (1974). The psychology of sex differences. Stanford, CA: Stanford University Press.

Petersen, A. C. (1976). Physical androgyny and cognitive functioning in adolescence. Developmental Psychology, 12, 524-533.

Strauss, E., \& Kinsbourne, M. (1981). Does age at menarche affect the ultimate level of verbal and spatial skills? Cortex, 17, 323-326.

Tanner, J. M. (1978). Fetus into man. Cambridge, MA: Harvard University Press.

WABE R, D. P. (1976). Sex differences in cognition: A function of maturation rate? Science, 192, 572-574.

WABER, D. P. (1977). Sex difference in mental abilities, hemispheric lateralization, and rate of physical growth at adolescence. Developmental Psychology, 13, 29-38.

WECHSLER, D. (1981). Wechsler Adult Intelligence Scale-Revised manual. New York: Psychological Corporation.

Witkin, H. A., Oltman, P. K., Raskin, E., \& KarP, S. A. (1971). A manual for the embedded figures tests. Palo Alto, CA: Consulting Psychologists Press.

Yarrow, M. R., Campbell, J. D., \& Burton, R. V. (1970). Recollections of childhood: A study of the retrospective method. Monographs of the Society for Research in Child Development, 35(5, Serial No. 138).

(Manuscript received for publication January 29, 1984.) 